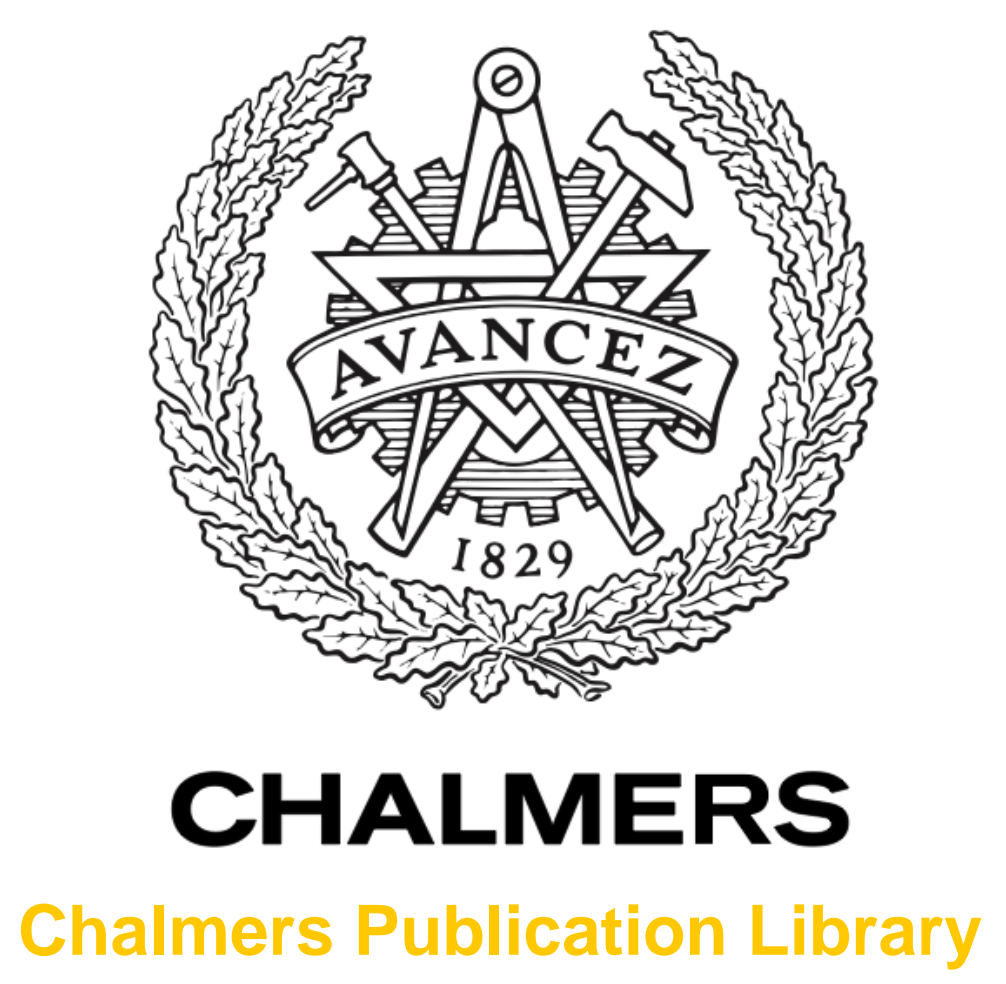

\title{
Light scattering by a cube: Accuracy limits of the discrete dipole approximation and the T-matrix method
}

This document has been downloaded from Chalmers Publication Library (CPL). It is the author's version of a work that was accepted for publication in:

Journal of Quantitative Spectroscopy and Radiative Transfer (ISSN: 0022-4073)

Citation for the published paper:

Yurkin, M. ; Kahnert, M. (2013) "Light scattering by a cube: Accuracy limits of the discrete dipole approximation and the T-matrix method". Journal of Quantitative Spectroscopy and Radiative Transfer, vol. 123 pp. 176-183.

http://dx.doi.org/10.1016/j.jqsrt.2012.10.001

Downloaded from: http://publications.lib.chalmers.se/publication/179405

Notice: Changes introduced as a result of publishing processes such as copy-editing and formatting may not be reflected in this document. For a definitive version of this work, please refer to the published source. Please note that access to the published version might require a subscription.

Chalmers Publication Library (CPL) offers the possibility of retrieving research publications produced at Chalmers University of Technology. It covers all types of publications: articles, dissertations, licentiate theses, masters theses, conference papers, reports etc. Since 2006 it is the official tool for Chalmers official publication statistics. To ensure that Chalmers research results are disseminated as widely as possible, an Open Access Policy has been adopted.

The CPL service is administrated and maintained by Chalmers Library. 


\title{
Light scattering by a cube: accuracy limits of the discrete dipole approximation and the T-matrix method
}

\author{
Maxim A. Yurkin, ${ }^{\mathrm{a}, \mathrm{b},{ }^{*}}$ Michael Kahnert ${ }^{\mathrm{c}, \mathrm{d}}$ \\ ${ }^{a}$ Institute of Chemical Kinetics and Combustion SB RAS, \\ Institutskaya Str. 3, 630090, Novosibirsk, Russia \\ ${ }^{b}$ Novosibirsk State University, Pirogova Str. 2, 630090, Novosibirsk, Russia \\ ${ }^{c}$ Swedish Meteorological and Hydrological Institute, \\ Folkborgsvägen 1, SE-601 76, Norrköping, Sweden \\ ${ }^{d}$ Chalmers University of Technology, Department of Earth and Space Science, \\ SE-412 96, Gothenburg, Sweden \\ *Corresponding author: yurkin@gmail.com
}

\begin{abstract}
We simulated light-scattering by small and wavelength-sized cubes with three largely different values of the refractive index using the discrete dipole approximation (DDA) and the T-matrix method. Our main goal was to push the accuracy of both methods to the limit. For the DDA we used an earlier developed extrapolation technique based on simulation results for different levels of discretization. For the T-matrix method we developed a procedure to estimate a confidence range for the simulated value, using results for different values of the truncation index (number of multipoles). In most cases this confidence range was reliable, enclosing the corresponding DDA result. We present benchmark results by both methods, including estimated uncertainties, for selected integral and angle-resolved scattering quantities. Estimated relative uncertainties of the DDA result are unprecedentedly small (from $10^{-7}$ to $10^{-3}$ ), while relative differences between the T-matrix and DDA results are larger (from $10^{-4}$ to 0.2 ) in accordance with estimated T-matrix uncertainties.
\end{abstract}

Keywords: discrete dipole approximation, light scattering simulation, T-matrix, cube, benchmark results 


\section{Introduction}

Light scattering by a cube is relevant for many practical applications, including powdered crystal samples [1], ice crystals in atmosphere [2], and metallic nanoparticles [3]. But even more often cubes are used in benchmark studies of light-scattering codes [4-7] to contrast with spheres and spheroids. Although a cube is a geometrically simple object, it is unexpectedly complicated in terms of light-scattering simulation. The Mie theory was proposed more than a century ago, and currently optical properties of spheres can be evaluated with machine precision [8]. By contrast, methods to rigorously evaluate lightscattering by cubes appeared only during the last two decades [4,9], and their accuracy is rarely discussed.

The first step in this direction was made by Fuchs [1] and Langbein [10] for cubes much smaller than the wavelength. They both used several terms of the eigenmode expansion of the surface-integral equation, limiting the accuracy of results to several percents. This approach was refined by Avelin et al. [11] making the errors less than 0.5\%. Optical properties of small cubes ( $k D$ up to 1.2 , where $k$ is free-space wavenumber and $D$ is the side length of the cube) were simulated using similar methods [12-14], again with several-percent accuracy, when compared against the finite-difference time-domain method (FDTD) [14]. The second step was made by Liou et al. [15] and later others [16,17] with geometrical-optics simulation of light scattering by cubes much larger than the wavelength.

Apparently, the first calculations for intermediate sized cubes $(k D \sim 5)$ were performed using the resolvent-kernel method [18] and the discrete dipole approximation (DDA) [9]. Both of these methods are based on the volume integral equation for the electric field [19]. Limited comparisons of the DDA with independent methods at that time [20,21] achieved relative differences between $1 \%$ and $10 \%$ depending on the refractive index.

Alternative methods, based on spherical basis functions were able to handle this problem only significantly later. In particular, the first application of the T-matrix method to simulate light-scattering by a cube was shown by Wriedt and Comberg [4]. Here and further we use this general term implying the extended boundary condition method. Kahnert et al. refined this approach by studying different methods for handling sharp edges [22] and explicitly employing cubical symmetries $[23,24]$. In comparison with the DDA the difference was several percents in angle-resolved quantities [23]. Recently, the T-matrix was applied to bi-anisotropic cubes [6], but the results were not compared to other methods. A cube was also 
handled by a modification of the T-matrix method [7], resulting in differences less than $10 \%$ when compared to the DDA.

While an accuracy of several percents is sufficient for most practical applications, better accuracy is desired for benchmark studies. Recently, Yurkin et al. [25] showed that the convergence of the DDA results with increasing discretization for a cube and other shapes, which can be exactly described as a set of cubes, is generally faster than for other shapes. Moreover, Richardson-type extrapolation was proposed for the DDA [26], which lead to extremely accurate results in combination with very fine discretization. In particular, for a cube with $k D=8$ and refractive index $m=1.5$ the relative error of the obtained extinction efficiency $Q_{\text {ext }}$ and maximum relative error in the phase function was estimated to be $2 \times 10^{-6}$ and $3 \times 10^{-5}$ respectively. This result was extended to other refractive indices - the relative error of absorption efficiency $Q_{\text {abs }}$ was estimated to be from $4 \times 10^{-7}$ to $2 \times 10^{-5}$ [27]. Moreover, for gold nanocubes $(D=10$ and $100 \mathrm{~nm})$ in the wavelength range $[400,800] \mathrm{nm}$ the errors of $Q_{\text {abs }}$ and $Q_{\text {sca }}$ were estimated to be less than $1 \%$ and $0.1 \%$ respectively [28]. However, the error estimate of the extrapolation technique is based on an empirical procedure; its reliability was only demonstrated for simulations with coarser discretization and hence larger errors [26]. Neither rigorous proves nor direct verifications of very small errors through comparisons with other light-scattering methods are available.

The goal of this paper is to extend the DDA results, claiming very good accuracy, to test them against the T-matrix method, and to provide benchmark numbers for several cubes, giving both the internal and independent (between-methods) error estimates. For that we use the symmetry-enhanced T-matrix code Tsym and push its accuracy to the limit, studying the dependence of the results on number of multipoles, i.e. on the T-matrix truncation index $n_{\text {cut }}$. The details of the methods and codes are given in Section 2, including the discussion of numerical exactness of both methods. A new procedure to analyze the T-matrix convergence curves is proposed in Section 3. Simulation results are shown and discussed in Section 4, and Section 5 concludes the paper.

\section{Methods}

\subsection{Test scatterers}

Since both codes applied here are mature and well-tested, we skip standard spherical benchmarks and focus on the main study object - a cube. Based on a previous study [27], we use three largely different refractive indices: $1.6+0.01 \mathrm{i}, 0.1+\mathrm{i}$, and $10+10 \mathrm{i}$, and two sizes: 
$k D=8$ and 0.1 (in total, 6 cubes). The $k D=8$ size is exactly the same as in [27], allowing us to reuse the corresponding DDA results. The T-matrix code could not produce satisfactory results for the cube with $k D=8$ and $m=10+10 \mathrm{i}$, hence we present only the DDA results for that particular case.

All considered cubes are oriented with edges parallel to the coordinate axes and illuminated by a plane wave, propagating along the $z$-axis. Angle-resolved quantities, such as the intensity $I=S_{11}$ (element of the Mueller matrix) and linear polarization ratio $P=-S_{21} / S_{11}$, are calculated in the $y z$-plane, characterized by a scattering angle $\theta$. All efficiencies $\left(Q_{\text {ext }}, Q_{\text {sca }}\right.$, $Q_{\text {abs }}$ ) are defined as corresponding cross sections divided by the geometrical cross section of a volume-equivalent sphere.

\subsection{The discrete dipole approximation}

As a DDA implementation we have used the code ADDA [29]. It is capable of running on a cluster of processors with distributed memory, which allowed us to use very fine discretization. For $k D=8$ cubes we reused the data from [27], which was calculated using ADDA v.0.78.2 running on the Dutch compute cluster LISA. ${ }^{1}$ The convergence criterion of the iterative solver $\varepsilon_{i t}$ was set to the default value $\left(10^{-5}\right)$ for $m=0.1+\mathrm{i}$ and $10+10 \mathrm{i}$ and to $10^{-10}$ for $m=1.6+0.01$. Filtered coupled dipoles (FCD) formulation of the DDA [27,30] and the default iterative solver (quasi-minimal residual method, QMR) was used for $m=10+10 \mathrm{i}$ and $1.6+0.01$. However, for $m=0.1+\mathrm{i}$ we used the default lattice-dispersion relation (LDR), since it resulted in smaller errors, and bi-conjugate-gradient stabilized method, since QMR encountered convergence problems for that particular case. For each cube 5 discretizations were used (characterized by the number of dipoles per cube side $n_{\mathrm{d}}=256,320,384,448$, and 512). Then the calculated dependencies of scattering quantities on $n_{\mathrm{d}}$ were extrapolated to infinite $n_{\mathrm{d}}$ using the procedure described in [26], producing both the value and estimate of its error (uncertainty). Computation wall clock times for the finest discretization $\left(n_{\mathrm{d}}=512\right)$ were from 0.4 (for $m=1.6+0.01$ ) to 6.5 hours (for $m=10+10 \mathrm{i}$ ) using 16 eight-core $(3.4 \mathrm{GHz}$ ) nodes.

For $k D=0.1$ cubes we used ADDA v.1.1 running on the computing cluster of the Supercomputing center of the Novosibirsk State University. ${ }^{2}$ The same settings - the FCD formulation, QMR iterative solver, and $\varepsilon_{\mathrm{it}}=10^{-10}$ - were used for all refractive indices. The

\footnotetext{
${ }^{1}$ http://www.sara.nl/systems/lisa/description

${ }^{2}$ http://www.nusc.ru/wiki/doku.php/doc/nusc/description
} 
discretizations and extrapolation procedure are the same as for $k D=8$. Differences in ADDA versions should not affect the simulation results. For this cube size simulation wall clock times are much smaller - from 2 (for $m=1.6+0.01$ ) to 30 minutes (for $m=10+10 \mathrm{i}$ ) for $n_{\mathrm{d}}=512$ using 16 eight-core $(2.5 \mathrm{GHz})$ nodes.

Finally, we note that the DDA is a rigorous ("numerically exact") method to solve Maxwell's equations [19], and the word "approximation" in its name is just a historical artifact. In particular, the DDA result converges to the exact value with refining discretization [25]. Moreover, the numerical stability of the DDA, e.g. measured by condition number of the corresponding system of linear equations, is almost independent of the discretization [27,31]. Therefore, convergence to an error on the order of a machine precision can be achieved with the current code just by adding (much) more computer power. Hence, the choice of the largest value of $n_{\mathrm{d}}$ (512) in this study is somewhat arbitrary. Further increase of $n_{\mathrm{d}}$ will improve the accuracy at the expense of larger computation time and required computer memory, which both scale as $\mathrm{O}\left(n_{d}^{3}\right)[29]$.

\subsection{The T-matrix method}

T-matrix computations have been performed with the code Tsym version $4.5[23,24]$. This program is applicable to non-axisymmetric, star-shaped homogeneous particles. It fully exploits group theoretical methods for particles with discrete geometrical symmetries; it uses the commutation relations of the T-matrix [23] to expedite computations, and it is, to the best of our knowledge, the only T-matrix code that exploits irreducible representations of point groups for reducing numerical problems due to ill-conditioned computations [24]. Cubes belong to the octahedral symmetry group $O_{h}$. The use of irreducible representations of cubic point groups is not yet fully operational in Tsym. However, dihedral point groups have been extensively used and tested in Tsym. Thus we used the dihedral symmetry group $D_{4 h}$, which is a subgroup of $O_{h}$, to expedite and numerically stabilize the T-matrix computations.

Computations were run in double precision on the Linux-based cluster Gimle of the National Supercomputer Centre in Linköping Sweden. ${ }^{3}$ Since the code is serial, it was run on a single $2.8 \mathrm{GHz}$ node. The computation time mainly depends on the truncation index $n_{\text {cut }}$ of the T-matrix, and on the number of quadrature points used in the numerical evaluation of the surface integrals in Waterman's extended boundary condition method. No attempt was made to optimize the computation time by minimizing the number of quadrature points in the

\footnotetext{
${ }^{3}$ http://www.nsc.liu.se/systems/gimle/
} 
different cases considered. Instead, the number of polar and azimuthal quadrature points was kept fixed; we used 800 quadrature points in the range $[0, \pi]$ for the polar angle, and 3200 quadrature points in the range $[0,2 \pi]$ for the azimuthal one. Note that, owing to the particle symmetries, the actual angular integration ranges are reduced in Tsym, see [22,23] for details. Thus, we used 400 quadrature points in the reduced polar range $[0, \pi / 2]$, and 400 quadrature points in the reduced azimuthal range $[0, \pi / 4]$. At a fixed value of $n_{\text {cut }}$, this choice gave stable results in all cases with respect to any further increase of the number of quadrature points. Typical values of $n_{\text {cut }}$ for which convergent results have been achieved vary from 7 to 45 . Within this range, the wall-clock time of the computations vary from 7 seconds to 1.8 hours. When comparing this to corresponding computation times of the DDA, it is important to emphasize that the information contents of T-matrix and DDA computations is not fully comparable. The T-matrix contains the complete information about a particle's scattering and absorption properties at a given wavelength. By contrast, DDA computations yield results for particles in one specific orientation. For instance, one can compute from the T-matrix, with very little extra investment of computation time, optical properties of ensembles of identical particles in random orientations, while DDA computations need to be repeated for each new angle of incidence.

The T-matrix method is also numerically exact in the sense that one can, in principle, produce arbitrarily exact results by increasing $n_{\text {cut }}$, the number of integration quadrature points, and the machine precision - see [32] for a detailed discussion. However, when working with a fixed machine precision (as was done in this study), the achievable precision is limited owing to numerical ill-conditioning and loss-of-accuracy problems. In particular, the condition number of the system of linear equations in the T-matrix method rapidly increases with $n_{\text {cut }}$, contrary to the one in the DDA with refining discretization (see Section 2.2). The severity of these problems depends on a number of factors ranging from size parameter and aspect ratio of the scatterer to the presence of small-scale roughness and edges and corners. Therefore, cubes are among the more challenging geometries in practical applications of the T-matrix method, while they are a "home game" for the DDA.

Finally, let us discuss the fundamental applicability of the T-matrix method to particles with $C^{0}$ surfaces (i.e. with sharp edges), such as a cube. For such surfaces, the surface integrals will have continuous, but not continuously differentiable integrands. However, continuity is sufficient for integrability. Thus, there are no principle mathematical problems in T-matrix calculations for particles with $C^{0}$ surfaces. We also know from numerical experiments that there are no principle problems in application of the T-matrix method to 
finite circular cylinders [33]. Critics may argue that the Gaussian quadrature scheme used in that T-matrix code does not include any points on the critical edge of the cylinder. However, the Tsym code used in our study has a different quadrature scheme for prisms (including cubes) that does include points on the critical edges of the top and bottom faces [23], and our experiences with the code do not indicate any fundamental problems related to particles with sharp edges. Perhaps even more important is the fact that a $C^{0}$ surface is an idealization that does not exist in nature. As remarked in [22], a "sharp edge" should be understood as a feature on the particle surface with a curvature radius much smaller than the wavelength. It has been demonstrated in that paper that the T-matrix results converge with decreasing radius of curvature $R_{\mathrm{c}}$. No indications of numerical instabilities were observed as $R_{\mathrm{c}}$ became much smaller than the wavelength.

All this indicates that there are no fundamental mathematical problems with sharp edges, but there are, to be sure, numerical challenges. The internal field near sharp edges and pointy corners has large gradients, i.e. the characteristic spatial scales are smaller than the particle size. Therefore, a large number of terms are needed in the spherical-harmonics expansions to accurately represent these fields. However, this is by no means limited to particles with sharp edges; we encounter the same problem in spheres perturbed with a smallscale surface roughness [34,35]. The surfaces of such particles are $C^{\infty}$, i.e. ultimately smooth. Yet to accurately describe the field induced in the small-scale surface features, a large number of expansion terms are required.

\section{Convergence of the T-matrix method}

Before one can meaningfully compare the DDA and T-matrix results, one should define what the T-matrix result is. While for the DDA this issue is (at least partly) addressed by the extrapolation technique (see Section 2.2), no straightforward approach is known for the Tmatrix method. The main problem is lack of uncertainty estimate associated with a computed value. Almost any T-matrix code incorporates some kind of an accuracy measure, which is usually based on the difference of results for two values of $n_{\text {cut }}$ [36-38]. However, to the best of our knowledge the relation between these measures and a real error, obtained by comparison with independent reference, has not been firmly established. This can be explained by the little relevance of this issue for simple (axisymmetric and moderate-sized) particles, for which the accuracy of the T-matrix method can be easily made good enough, taking large enough $n_{\text {cut }}$. By contrast, for non-axisymmetric particles references with welldefined accuracy are lacking. 
The deficiencies of existing error measures has been recently discussed [37,38]. To illustrate this issue, we present two typical convergence plots of the T-matrix results with varying $n_{\text {cut }}$ in Fig. 1 . The common feature of both parts of these graphs is that larger values of $n_{\text {cut }}$ do not necessarily imply better accuracy. There always exists an optimal range of $n_{\text {cut }}$, where the variation of values is smallest, such as the range [15;40]. However, the convergence behavior can be different. In Fig. 1(b) the computed value oscillates around a certain level and the difference between, e.g., $n_{\text {cut }}$ and $n_{\text {cut }}-3$ provides an adequate estimate of the uncertainty. By contrast, Fig. 1(a) depicts an almost monotonic convergence, which is truncated by numerical instability at larger $n_{\text {cut }}$ (this instability is analyzed in [32]). In particular, the difference between two values of $n_{\text {cut }}$ can be much smaller than the real error. All other Tmatrix convergence curves (data not shown) fall into one of these two categories - with or without monotonic convergence.

We note that this convergence curve is not a series in a standard sense, because incrementing $n_{\text {cut }}$ not only adds a new term to the sum, but also modifies all existing terms. Moreover, contrary to the DDA (see Section 2.2) throwing in more computer power will not help. Therefore, the full convergence curve defines the best possible result achievable with the current code (see Section 2.2). The only way to improve it is to modify the code, e.g. by using better working precision. The most rigorous approach would be to compare the whole Tmatrix convergence curve against the DDA results. This is done in Fig. 1, showing good agreement within the variation of the T-matrix values. However, such qualitative comparison is not practical for a large number of computed values, including angle-resolved ones. Therefore, we propose the following empirical procedure to compress the T-matrix convergence curve into the confidence interval, which defines the T-matrix result.

- First, we manually choose the range of $n_{\text {cut }}$, trying both to keep the variation of values close to the minimum and to maximize the range size. The particular choices for all studied cubes are summarized in Table 1.

- Second, we fit the truncated curve by $f(x)=a+b x^{-c}$. This function is somewhat arbitrary, but we also tried an exponential one (data not shown) resulting in consistently worse fits.

- The fit is considered successful if the minimum of the residual sum of squares (RSS) is reached for $0<c<\infty$, and the null hypotheses $(c=0$ or $c=\infty)$ can be rejected with a $p$ value of 0.05 based on Fisher's criterion, i.e. the ratio of RSS for the null hypothesis to the minimal one is larger than the appropriate quantile of Fisher's distribution. Then we determine the 95\%-confidence interval for $f(100)$ based on the upper bound for RSS. Using $f(100)$ instead of $a=f(\infty)$ is more robust for small $c$. This confidence interval is 
generally asymmetric (up to 20\%) about the best-fit value, but that is negligible considering the approximate nature of the whole procedure. Thus in the following we present the best fit of $f(100) \pm$ half of the confidence range.

- If the fit is unsuccessful (see above) we instead fit the data by a simpler function, corresponding to $c=0$ (constant) or $c=\infty$ (constant with the data point for the smallest $n_{\text {cut }}$ omitted). Then the best-fit value is just the mean of the data, while the $95 \%$ confidence width is determined from the standard deviation multiplied by appropriate quantile of Student's distribution.

An illustrative application of this procedure is presented in Fig. 1, which shows both the fitted curve and the obtained confidence interval. We note that exactly the same procedure applies to both convergence cases. The T-matrix result shows good correspondence with the DDA results. We stress, however, that the proposed procedure is mostly a convenient technique to quantitatively compare T-matrix results with other methods. While in certain cases it works surprisingly well [Fig. 1(a)], it can also significantly under- or overestimate the real error. The quality of this procedure for cubes can be judged based on results in Section 4 . However, further testing (and probably refinement) of this procedure is definitely required before it can be broadly applied.

\section{Comparison between the methods}

In this section we present the final processed results for the cubes, obtained using extrapolation techniques described in Sections 2.2 and 3 for the DDA and the T-matrix method, respectively. First we consider $k D=0.1$ cubes. Since the angular dependence of both $I$ and $P$ is trivial for such small particles, we show only the results for efficiencies in Table 2. The internal error estimates of the DDA results are 2-4 orders of magnitude smaller than those of the T-matrix results. Hence the DDA results can be considered as a reliable reference, even if its error estimates are only correct by an order of magnitude. Relative differences between the DDA and T-matrix results are between $0.03 \%$ and $0.5 \%$, except $2 \%$ difference for $Q_{\text {sca }}$ for $m=0.1+\mathrm{i}$. These differences are smaller (up to 30 times) than the T-matrix error estimate except for $Q_{\text {sca }}$ for $m=10+10 \mathrm{i}$, where it is 2 times larger.

For $k D=8$ cubes we summarize benchmark results in Table 3, showing both efficiencies and several particular values of $I$ and $P$. Full angle-dependencies of $I$ and $P$, computed with the DDA, are shown in Fig. 2, while the corresponding difference of the Tmatrix and DDA results is presented in Fig. 3 together with error estimates of each method. The overall conclusions are the same as for $k D=0.1$. Differences between the T-matrix and 
DDA results are from $0.01 \%$ (excluding occasional smaller values) to $0.3 \%$ for $m=1.6+0.01 \mathrm{i}$ and from $0.3 \%$ to $15 \%$ for $m=0.1+\mathrm{i}$ (efficiencies agree within $3 \%$ )

Considering the DDA result as a reference, we can deduce a strong correlation between the estimated and real errors of the T-matrix results. However, for some scattering quantities the estimate is too conservative, being more than 10 times larger than the real error. For other cases, by contrast, the estimate is up to 4 times smaller than the real error. This applies, in particular, to $Q_{\text {sca }}$ and $Q_{\text {abs }}$ for $m=0.1+\mathrm{i}$ and to $P$ near the back-scattering direction. To analyze this behavior we present one particular case in Fig. 4. The default fit procedure, as defined in Section 3, leads to confidence interval (denoted by the square symbol) that significantly disagrees with the DDA result. However, the disagreement with the DDA is not that obvious if we consider the whole convergence curve. For instance, if the $n_{\text {cut }}$ range used for fitting is decreased to [20;35], which can be justified by larger variation of the T-matrix results at the ends of the original range, the confidence interval moves closer to and broadens to include the DDA result (see the diamond symbol). Thus, we conclude that this and other (data not shown) underestimation of the real error is caused by imperfection of the employed simple fitting procedure.

\section{Conclusion}

We have simulated light-scattering by several cubes with the DDA and T-matrix method, pushing the accuracy of both methods to the limit. For the DDA we used an earlier developed extrapolation technique based on simulation results for different levels of discretization. It allowed us to present unprecedentedly accurate benchmark results with estimated relative uncertainty from $10^{-7}$ to $10^{-3}$ depending on cube size and refractive index, as well as on the particular scattering quantity of interest.

For the T-matrix method we analyzed convergence curves (versus $n_{\text {cut }}$ ) and showed that some cases feature almost monotonous convergence. Based on this we proposed a simple procedure to fit this curve by a power function, which allowed us to compress the whole convergence curve into a confidence range (i.e. a certain value and its uncertainty estimate). Comparison of the DDA and T-matrix results showed that the obtained estimate is, overall, reliable, although an underestimation of the real error up to a factor of four was obtained in rare cases. This warrants a further study of this procedure for other scattering problems, especially those for which the T-matrix method suffers numerical instability (scatterers with large sizes or aspect ratios, surface roughness, or edges). 
The confidence intervals determined for the T-matrix and DDA results can be interpreted as a measure that reflects the numerical behavior of either method. While DDA computations progressively improve as the number of dipoles is increased, the accuracy of the T-matrix method is often limited by the oscillating behavior of the results as a function of $n_{\text {cut }}$, as well as by the divergence of the results at very large values of $n_{\text {cut }}$. Both the proposed error estimate of the T-matrix method and its difference against the DDA results showed relative errors from $10^{-4}$ to 0.2 . Moreover, T-matrix error estimate was always from 100 to $10^{4}$ times larger than the DDA error estimate. Although we could not directly verify the accuracy claimed by the DDA, even the less remarkable agreement between the two methods is unprecedently good in some cases. Thus we believe that the presented benchmark results would be useful for developers of light scattering codes, as well as for those who apply these codes to cubes in practical applications.

Finally, we note that a standard approach for light-scattering benchmark studies is to evaluate the accuracy of the DDA versus the reference provided by the T-matrix method [5,39]. By contrast, we started with equal attitude to both methods and ended up with using the DDA as the reference to evaluate the accuracy of the T-matrix method. This outcome is partly due to the fact that with ever-increasing computational resources it is easier to blindly throw in more computer power than to increase the working precision of the existent code or to develop preconditioning strategies (at least for a small number of benchmark results). While both cubical shape and fixed orientation contribute in favor of the DDA in this respect, the DDA has also been shown superior for large smooth (and even axisymmetric) particles with small optical contrast. For instance, the T-matrix and similar methods based on spherical-harmonics expansion have not been able so far to handle red blood cells with size parameters larger than 38 [37,40] due to its biconcave shape. By contrast, DDA simulation for a sphere with size parameter 320 and refractive index 1.05 was demonstrated [29] albeit using a large computer cluster. Similar DDA applicability range is expected for (moderately) nonspherical particles, e.g. spheroids, which is still to be matched by the T-matrix or similar methods.

On the other hand, the T-matrix approach lends itself more easily to other complex morphologies, e.g., particles with small-scale surface roughness, especially when the surface perturbations have sub-wavelength scale. Novel numerical schemes for performing T-matrix computations have recently been reported that substantially extend the size parameter range while reducing CPU time requirements for such morphologies [41]. By contrast, the DDA computations require a very large number of dipoles to resolve such surface features, which 
can result in high CPU time requirements and more severe limitations in the range of accessible size parameters [34]. Therefore, further development of both methods, including numerical aspects of the corresponding codes, is definitely an important research topic.

\section{Acknowledgements}

We would like to thank Vladimir Schmidt and Tom Rother for helpful discussions, and anonymous reviewers for helpful comments. M. Yurkin acknowledges support of the program of the Russian Government "Research and educational personnel of innovative Russia" (contracts P422, P1039, and 14.740.11.0921), by grant from Russian Government 11.G34.31.0034, and by Russian Foundation of Basic Research (grant 12-04-00737-a). M. Kahnert acknowledges funding from the Swedish Research Council (Vetenskapsrådet) under projects 621-2008-4387 and 621-2011-3346.

\section{References}

[1] Fuchs R. Theory of the optical properties of ionic crystal cubes. Phys. Rev. B 1975;11:1732-1740.

[2] Kokhanovsky AA. Modeling of light depolarization by cubic and hexagonal particles in noctilucent clouds. Atmos. Res. 2006;79:175-181.

[3] Lu X, Rycenga M, Skrabalak SE, Wiley B, Xia Y. Chemical synthesis of novel plasmonic nanoparticles. Annu. Rev. Phys. Chem. 2009;60:167-192.

[4] Wriedt T, Comberg U. Comparison of computational scattering methods. J. Quant. Spectrosc. Radiat. Transfer 1998;60:411-423.

[5] Penttila A, Zubko E, Lumme K, Muinonen K, Yurkin MA, Draine BT, et al. Comparison between discrete dipole implementations and exact techniques. J. Quant. Spectrosc. Radiat. Transfer 2007;106:417-436.

[6] Schmidt V, Wriedt T. T-matrix method for biaxial anisotropic particles. J. Quant. Spectrosc. Radiat. Transfer 2009;110:1392-1397.

[7] Petrov D, Shkuratov Y, Videen G. An analytical solution to the light scattering from cube-like particles using Sh-matrices. J. Quant. Spectrosc. Radiat. Transfer 2010;111:474482.

[8] Bohren CF, Huffman DR. Absorption and Scattering of Light by Small Particles. New York: Wiley; 1983. 
[9] Flatau PJ, Stephens GL, Draine BT. Light-scattering by rectangular solids in the discrete-dipole approximation - a new algorithm exploiting the block-Toeplitz structure. J. Opt. Soc. Am. A 1990;7:593-600.

[10] Langbein D. Normal modes at small cubes and rectangular particles. J. Phys. A 1976;9:627-644.

[11] Avelin J, Sharma R, Hanninen I, Sihvola AH. Polarizability analysis of cubical and square-shaped dielectric scatterers. IEEE Trans. Antennas Propag. 2001;49:451-457.

[12] Moheb H, Shafai L. Numerical solution of integral equations for dielectric objects of prismatic shapes. IEEE Trans. Antennas Propag. 1991;39:758-766.

[13] Povinelli MJ, Harrington RF. A volume and surface source integral formulation for electromagnetic scattering from printed-circuit antennas. IEEE Trans. Magn. 1991;27:42714274.

[14] Inoue T, Inagaki N, Kikuma N. Modal polarization current model method and its application to scattering by dielectric bodies. Electr. Commun. Japan Part 1 2003;86:25-34.

[15] Liou KN, Cai Q, Pollack JB, Cuzzi JN. Light scattering by randomly oriented cubes and parallelepipeds. Appl. Opt. 1983;22:3001-3008.

[16] Grynko Y, Shkuratov Y. Scattering matrix calculated in geometric optics approximation for semitransparent particles faceted with various shapes. J. Quant. Spectrosc. Radiat. Transfer 2003;78:319-340.

[17] Hesse E, Macke A, Havemann S, Baran AJ, Ulanowski Z, Kaye PH. Modelling diffraction by facetted particles. J. Quant. Spectrosc. Radiat. Transfer 2012;113:342-347.

[18] Kattawar GW, Hu C-R, Parkin ME, Herb P. Mueller matrix calculations for dielectric cubes: comparison with experiments. Appl. Opt. 1987;26:4174-4180.

[19] Yurkin MA, Hoekstra AG. The discrete dipole approximation: an overview and recent developments. J. Quant. Spectrosc. Radiat. Transfer 2007;106:558-589.

[20] Hage JI, Greenberg JM, Wang RT. Scattering from arbitrarily shaped particles: theory and experiment. Appl. Opt. 1991;30:1141-1152.

[21] Rouleau F, Martin PG. A new method to calculate the extinction properties of irregularly shaped particles. Astrophys. J. 1993;414:803-814.

[22] Kahnert FM, Stamnes JJ, Stamnes K. Application of the extended boundary condition method to particles with sharp edges: a comparison of two surface integration approaches. Appl. Opt. 2001;40:3101-3109. 
[23] Kahnert FM, Stamnes JJ, Stamnes K. Application of the extended boundary condition method to homogeneous particles with point-group symmetries. Appl. Opt. 2001;40:3110 3123.

[24] Kahnert M. Irreducible representations of finite groups in the T-matrix formulation of the electromagnetic scattering problem. J. Opt. Soc. Am. A 2005;22:1187-1199.

[25] Yurkin MA, Maltsev VP, Hoekstra AG. Convergence of the discrete dipole approximation. I. Theoretical analysis. J. Opt. Soc. Am. A 2006;23:2578-2591.

[26] Yurkin MA, Maltsev VP, Hoekstra AG. Convergence of the discrete dipole approximation. II. An extrapolation technique to increase the accuracy. J. Opt. Soc. Am. A 2006;23:2592-2601.

[27] Yurkin MA, Min M, Hoekstra AG. Application of the discrete dipole approximation to very large refractive indices: Filtered coupled dipoles revived. Phys. Rev. E 2010;82:036703.

[28] Yurkin MA, de Kanter D, Hoekstra AG. Accuracy of the discrete dipole approximation for simulation of optical properties of gold nanoparticles. J. Nanophoton. 2010;4:041585.

[29] Yurkin MA, Hoekstra AG. The discrete-dipole-approximation code ADDA: capabilities and known limitations. J. Quant. Spectrosc. Radiat. Transfer 2011;112:22342247.

[30] Piller NB, Martin OJF. Increasing the performance of the coupled-dipole approximation: A spectral approach. IEEE Trans. Antennas Propag. 1998;46:1126-1137.

[31] Rahola J. Solution of dense systems of linear equations in the discrete-dipole approximation. SIAM J. Sci. Comp. 1996;17:78-89.

[32] Somerville WRC, Auguié B, Le Ru EC. Severe loss of precision in calculations of Tmatrix integrals. J. Quant. Spectrosc. Radiat. Transfer 2012;113:524-535.

[33] Mishchenko MI, Travis LD, Macke A. Scattering of light by polydisperse, randomly oriented, finite circular cylinders. Appl. Opt. 1996;35:4927-4940.

[34] Kahnert M, Nousiainen T, Thomas MA, Tyynelä J. Light scattering by particles with small-scale surface roughness: Comparison of four classes of model geometries. J. Quant. Spectrosc. Radiat. Transfer. Forthcoming. doi: 10.1016/j.jqsrt.2012.03.017.

[35] Kahnert M. T-matrix computations for particles with high-order finite symmetries. J. Quant. Spectrosc. Radiat. Transfer. this issue. 
[36] Mishchenko MI. Light-scattering by size-shape distributions of randomly oriented axially-symmetrical particles of a size comparable to a wavelength. Appl. Opt. 1993;32:46524666.

[37] Hellmers J, Schmidt V, Wriedt T. Improving the numerical stability of T-matrix light scattering calculations for extreme particle shapes using the nullfield method with discrete sources. J. Quant. Spectrosc. Radiat. Transfer 2011;112:1679-1686.

[38] Schmidt K, Yurkin MA, Kahnert M. A case study on the reciprocity in light scattering computations. Opt. Express 2012;20:23253-23274.

[39] Hovenier JW, Lumme K, Mishchenko MI, Voshchinnikov NV, Mackowski DW, Rahola J. Computations of scattering matrices of four types of non-spherical particles using diverse methods. J. Quant. Spectrosc. Radiat. Transfer 1996;55:695-705.

[40] Gilev KV, Eremina E, Yurkin MA, Maltsev VP. Comparison of the discrete dipole approximation and the discrete source method for simulation of light scattering by red blood cells. Opt. Express 2010;18:5681-5690.

[41] Kahnert M, Rother T. Modeling optical properties of particles with small-scale surface roughness: combination of group theory with a perturbation approach. Opt. Express 2011;19:11138-11151. 
Table 1. Ranges of $n_{\text {cut }}$ used for processing T-matrix convergence curves.

\begin{tabular}{|ll|l|l|l|}
\hline$k D$ & $m$ & \multicolumn{1}{|c|}{$1.6+0.01 \mathrm{i}$} & \multicolumn{1}{|c|}{$0.1+\mathrm{i}$} & \multicolumn{1}{|c|}{$10+10 \mathrm{i}$} \\
\hline 0.1 & & {$[7 ; 30]$} & {$[11 ; 30]$} & {$[10 ; 30]$} \\
\hline 8 & & {$[15 ; 40]$} & {$[20 ; 45]$} & - \\
\hline
\end{tabular}

Table 2. Benchmark results for $k D=0.1$ cubes. Uncertainties $( \pm)$ are in the last shown digits of the corresponding value and have nominal confidence level of $95 \%$.

\begin{tabular}{|l|l|l|l|l|}
\hline & $m$ & \multicolumn{1}{|c|}{$1.6+0.01 \mathrm{i}$} & \multicolumn{1}{c|}{$0.1+\mathrm{i}$} & $10+10 \mathrm{i}$ \\
\hline$Q_{\text {ext }}$ & DDA & $0.0012368241 \pm 1$ & $0.206751 \pm 1$ & $0.0122143 \pm 4$ \\
\hline & T-matrix & $0.0012372 \pm 9$ & $0.2069 \pm 33$ & $0.01216 \pm 7$ \\
\hline$Q_{\text {sca }}$ & DDA & $(4.85770 \pm 3) \times 10^{-6}$ & $(4.592 \pm 1) \times 10^{-5}$ & $(5.86192 \pm 5) \times 10^{-5}$ \\
\hline & T-matrix & $(4.850 \pm 27) \times 10^{-6}$ & $(4.67 \pm 15) \times 10^{-5}$ & $(5.849 \pm 5) \times 10^{-5}$ \\
\hline$Q_{\text {abs }}$ & DDA & $0.0012319664 \pm 1$ & $0.206705 \pm 1$ & $0.0121557 \pm 4$ \\
\hline & T-matrix & $0.0012324 \pm 10$ & $0.2068 \pm 33$ & $0.01211 \pm 7$ \\
\hline
\end{tabular}

Table 3. Same as Table 2 but for $k D=8$ cubes. The T-matrix method failed for $m=10+10 \mathrm{i}$.

\begin{tabular}{|l|l|l|l|l|}
\hline & $\mathrm{m}$ & \multicolumn{1}{|c|}{$1.6+0.01 \mathrm{i}$} & \multicolumn{1}{c|}{$0.1+\mathrm{i}$} & \multicolumn{1}{c|}{$10+10 \mathrm{i}$} \\
\hline$Q_{\text {ext }}$ & DDA & $4.2480442 \pm 3$ & $2.816375 \pm 7$ & $2.48217 \pm 7$ \\
\hline & T-matrix & $4.24775 \pm 33$ & $2.795 \pm 26$ & - \\
\hline$Q_{\text {sca }}$ & DDA & $3.9715646 \pm 3$ & $2.229316 \pm 7$ & $2.11276 \pm 1$ \\
\hline & T-matrix & $3.97156 \pm 24$ & $2.251 \pm 16$ & - \\
\hline$Q_{\text {abs }}$ & DDA & $0.2764796 \pm 1$ & $0.58706 \pm 1$ & $0.36941 \pm 1$ \\
\hline & T-matrix & $0.2757 \pm 7$ & $0.573 \pm 5$ & - \\
\hline$I\left(0^{\circ}\right)$ & DDA & $725.8766 \pm 2$ & $356.391 \pm 1$ & $234.86 \pm 2$ \\
\hline & T-matrix & $725.81 \pm 13$ & $350 \pm 8$ & - \\
\hline$I\left(90^{\circ}\right)$ & DDA & $5.071924 \pm 2$ & $2.5343 \pm 5$ & $5.4350 \pm 1$ \\
\hline & T-matrix & $5.087 \pm 13$ & $2.523 \pm 79$ & - \\
\hline$I\left(180^{\circ}\right)$ & DDA & $3.72126 \pm 1$ & $72.982 \pm 7$ & $75.095 \pm 7$ \\
\hline & T-matrix & $3.725 \pm 15$ & $78.6 \pm 55$ & - \\
\hline$P\left(90^{\circ}\right)$ & DDA & $-0.2574261 \pm 2$ & $0.7871 \pm 3$ & $0.1976 \pm 3$ \\
\hline & T-matrix & $-0.2569 \pm 19$ & $0.838 \pm 48$ & - \\
\hline
\end{tabular}




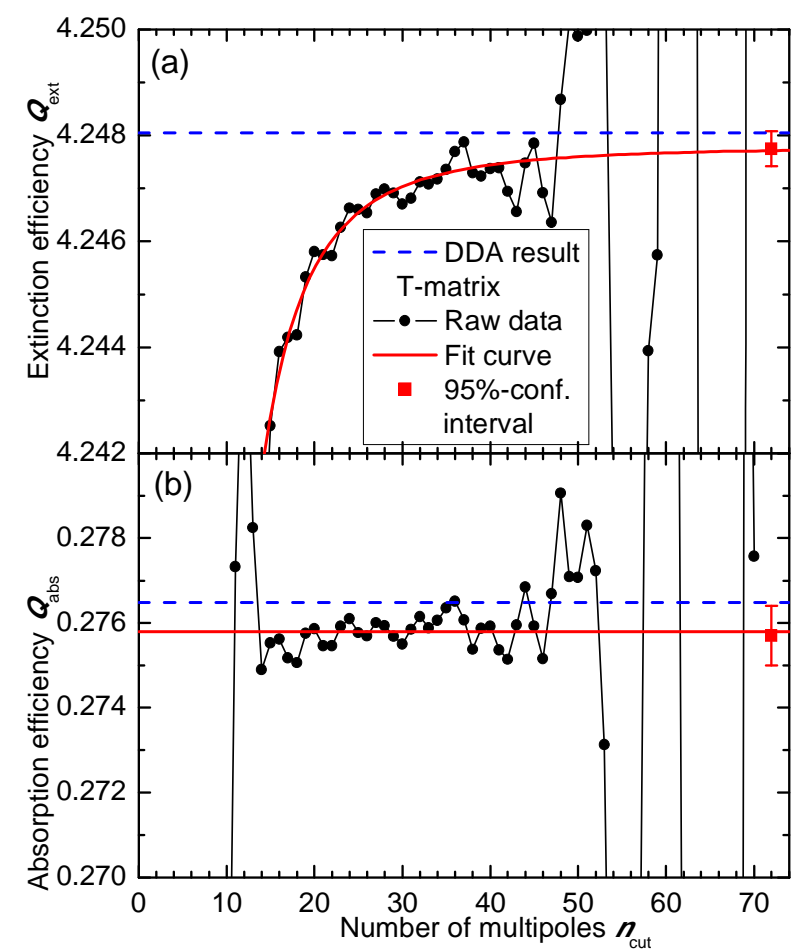

Fig. 1. Dependence of $Q_{\text {ext }}$ (a) and $Q_{\text {abs }}$ (b) on $n_{\text {cut }}$ for a cube with $k D=8$ and $m=1.6+0.01$ i computed with the T-matrix method. Fitted curves and estimated confidence ranges are also shown (based on data for $15 \leq n_{\text {cut }} \leq 40$, see text). DDA results (extrapolated) are shown for reference; their estimated uncertainty is less than the line width (see Table 2). 


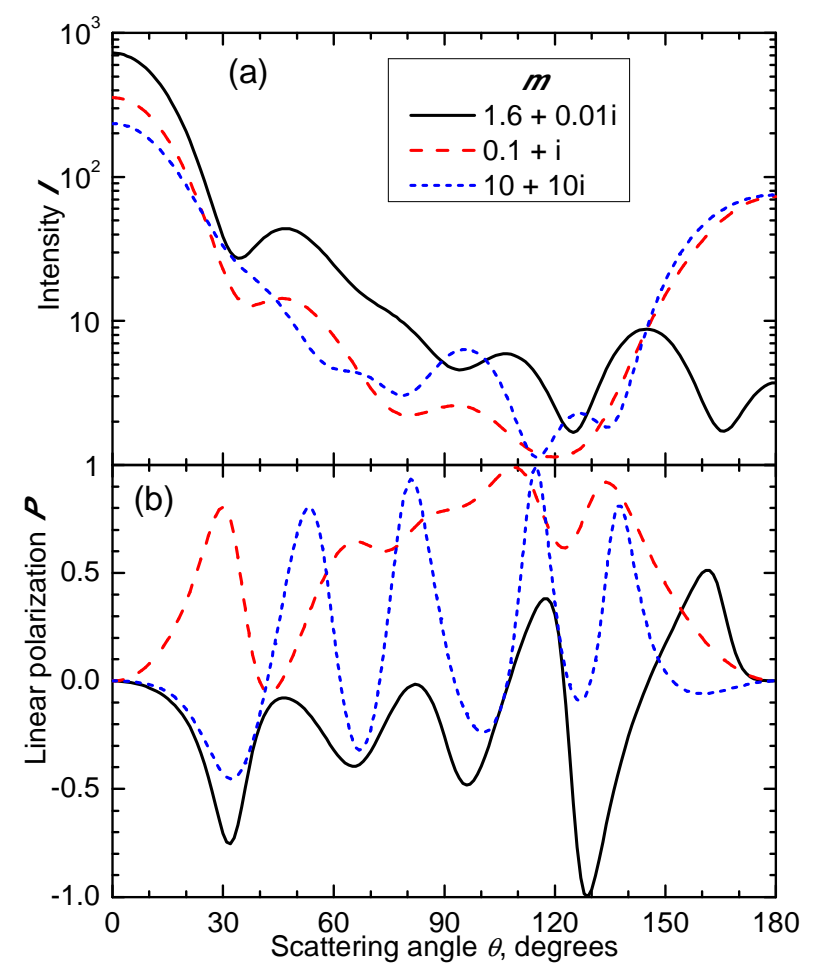

Fig. 2. DDA results for $I$ (a, logarithmic scale) and $P(\mathrm{~b})$ of $k D=8$ cubes. The T-matrix results are omitted for clarity of presentation. See Fig. 3 for differences. 


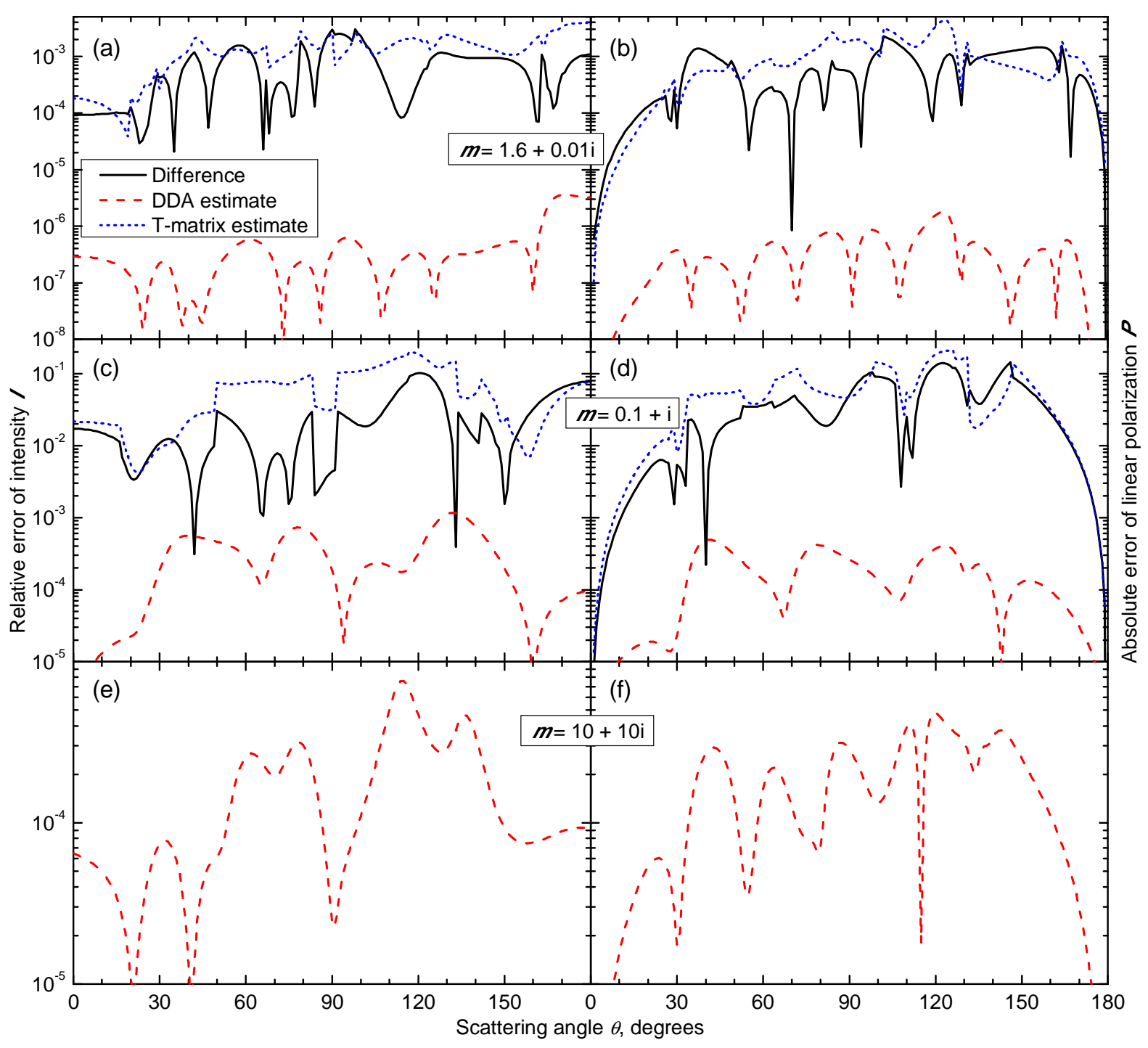

Fig. 3. Comparison between DDA and T-matrix results in terms of relative difference of $I$ (left row) and absolute difference of $P$ (right row) for $k D=8$ cubes with three refractive indices. Together with differences, internal error estimates of each method are shown. The T-matrix method failed for $m=10+10 \mathrm{i}$, so only the DDA error estimate is shown in this case. 


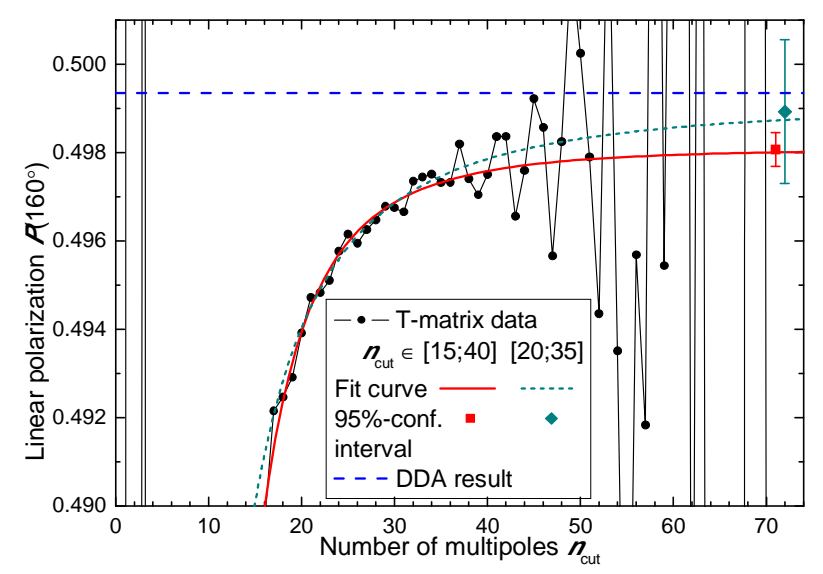

Fig. 4. Dependence of $P\left(160^{\circ}\right)$ on $n_{\text {cut }}$ for a cube with $k D=8$ and $m=1.6+0.01$ i computed with the T-matrix method. Fitted curves and estimated confidence ranges are shown, derived from two subsets of data $-n_{\text {cut }} \in[15,40]$ (default) and $[20,35]$. The DDA result (extrapolated) is shown for reference; its estimated uncertainty is less than line width (see Fig. 3). 